\title{
Transient state temperature distribution in a cylindrical electrical conductor : non-linear effects
}

\author{
A. Jordan, M. Benmouna, A. Borucki and F. Bouayed \\ Institut National de l'Enseignement Supérieur, Département de Physique, B.P. 119, Tlemcen, Algérie
}

(Reçu le 4 décembre 1984, révisé le 22 octobre 1985, accepté le 23 décembre 1985)

\begin{abstract}
Résumé. - Nous appliquons la méthode de linéarisation optimale au problème de transfert de chaleur dans un conducteur cylindrique en régime transitoire et dans les cas où la conductivité thermique et la chaleur spécifique varient linéairement avec la température. Nous obtenons un bon accord entre la résolution de l'équation non linéaire et la solution analytique de l'équation optimale linéaire correspondante. Nous considérons aussi bien le cas d'un nombre de Biot petit que celui d'un nombre de Biot supérieur à l'unité.
\end{abstract}

\begin{abstract}
The optimal linearization method is used to investigate the time dependence of the temperature in a cylindrical conductor in the case where the thermal conductivity and the specific heat are linear functions of the temperature. The agreement between the numerical resolution of the non linear equation and the linearized version is found to be good. Cases of small and large values of Biot's number are considered.
\end{abstract}

\section{Introduction.}

In a preceding paper [1] we have solved the problem of steady state heat transfer in a cylindrical conductor in the case where the thermal conductivity $\lambda(T)$ and the electrical resistivity $\rho(T)$ are linear functions of the temperature. The heat transfer in this case is governed by a non-linear differential equation which we solved by two different ways. The first way was to use a numerical scheme based on the Range-Kutta method which led to an exact solution. The second way was to approximate the non-linear differential equation by an optimal linear differential equation containing an optimal thermal conductivity $\lambda_{\text {op }}$ which is independent of $T$.

In the case of small values of the Biot's [8] number, the effect of non-linearity due to $\lambda(T)$ was found to be small even when the amplitude of the non-linearity is deliberately increased well above the known levels for classical electrical conductors such as aluminium and copper. In this paper, we intend to extend this investigation to the transient state which characterizes the rise of temperature with time when the electrical current is switched on. Here also we consider linear variations of $\lambda(T)$ and $\rho(T)$ with temperature. In fact we consider another important case when the specific heat $c(T)\left[\mathrm{J} \mathrm{kg}^{-1} \mathrm{~K}^{-1}\right]$ is also a linear function of temperature. All these cases, beside the fact that they represent actual problems from a practical point of view $[2,3]$, they lead to interesting non-linear partial differential equations which can be solved by using our optimal linearization method. The heat transfer equation for an arbitrary coordinate system and in the case where all physical parameters are functions of $T$, is :

$$
\nabla \cdot[\lambda(T) \nabla T]-c(T) \delta(T) \frac{\partial T}{\partial t}=-q(\mathbf{r}, T),
$$

where $\delta(T)\left[\mathrm{kg} \mathrm{m}^{-3}\right]$ is the mass density, $q(\mathbf{r}, t)$ is the rate of internal production, $\mathbf{r}$ is the space parameter and $t$ the time.

Here $T$ represents actually the rise of temperature above the ambient temperature $T_{\mathrm{a}}$. In this calculation, we assume that $\delta(T)=\delta_{0}$ is a constant and that the internal heat production is given by :

$$
q(\mathbf{r}, T)=\rho(T) j^{2},
$$

where $j$ is the direct current density which is chosen to be constant. If we choose a constant voltage $E[\mathrm{~V} / \mathrm{m}]$, the heat production becomes $q(\mathbf{r}, t)=E^{2} / \rho(T)$ and we can apply the same calculation as for equation (2). This was done in the case of a small value of the Biot's number and the difference was found to be negligible. 
The procedure used here to solve the transient heat problem is a straightforward generalization of the steady state calculation [1].

The numerical resolution is a combination of the finite difference method for the time differentiation and the Range-Kutta method for the space differentiation. The approximate resolution is an extension of the method of optimal linearization described in reference [1] to the time dependent problem.

\section{Resolution of the transient state heat equation.}

For simplicity but without loss of generality, we consider the case of an infinitely long, cylindrical and isotropic conductor. In all cases, we shall consider that $\rho(T)=\rho_{0}(1+\alpha T)$ unless when it is specified otherwise explicitly. We shall examine the non-linear effects due to $\lambda(T)$ and $c(T)$ separately.

2.1 THE CASE WHERE $\lambda(T)=\lambda_{0}\left(1+\alpha^{\prime} T\right)$ AND $c(T)=c_{0}$. - In this case, the heat transfer equation becomes:

$$
\begin{aligned}
\lambda_{0}\left(1+\alpha^{\prime} T\right)\left[\frac{\partial^{2} T}{\partial r^{2}}+\frac{1}{r} \frac{\partial T}{\partial r}\right] & +\lambda_{0} \alpha^{\prime}\left(\frac{\partial T}{\partial r}\right)^{2}- \\
-\delta_{0} c_{0} \frac{\partial T}{\partial t} & =-\rho_{0}(1+\alpha T) j^{2}
\end{aligned}
$$

To solve this equation, one needs two boundary conditions and an initial condition which are chosen as follows :

$$
\begin{gathered}
\left.\frac{\partial T}{\partial r}\right|_{r=0}=0, \\
\left.\lambda(T) \frac{\partial T}{\partial r}\right|_{r=r_{0}}=-\varepsilon T\left(r_{0}\right), \\
T(r, t=0)=0, \quad 0 \leqslant r \leqslant r_{0} .
\end{gathered}
$$

where $r_{0}$ is the radius of the conductor, and $\varepsilon$ the convective transfer coefficient.

The optimal linearization method consists of writing the following linear equation :

$$
\lambda_{\mathrm{op}}\left[\frac{\partial^{2} T}{\partial r^{2}}+\frac{1}{r} \frac{\partial T}{\partial r}\right]-c_{0} \delta_{0} \frac{\partial T}{\partial t}=-\rho_{0}(1+\alpha T) j^{2},
$$

which involves an optimal constant thermal conductivity $\lambda_{\text {op }}$. This parameter is calculated using a straightforward generalization of the optimal linearization method described in reference [1] which takes into account the time dependence of the temperature distribution [4]. We obtain :

$$
\lambda_{\mathrm{op}}=\lambda_{0}\left(1+\alpha^{\prime} E\right),
$$

where $E$ is given by :

$$
E=\frac{\int_{0}^{r_{0}} \int_{0}^{t_{1}} \mathrm{~d} r \mathrm{~d} t T\left[\frac{\partial^{2} T}{\partial r^{2}}+\frac{1}{r} \frac{\partial T}{\partial r}\right]^{2}+\int_{0}^{r_{0}} \int_{0}^{t_{1}} \mathrm{~d} r \mathrm{~d} t\left(\frac{\partial T}{\partial r}\right)^{2}\left[\frac{\partial^{2} T}{\partial r^{2}}+\frac{1}{r} \frac{\partial T}{\partial r}\right]}{\int_{0}^{r_{0}} \int_{0}^{t_{1}} \mathrm{~d} r \mathrm{~d} t\left[\frac{\partial^{2} T}{\partial r^{2}}+\frac{1}{r} \frac{\partial T}{\partial r}\right]^{2}},
$$

where $t_{1}$ is the time at which the steady state regime is approximately reached. We shall clarify this point later. In any case we assume that for our physical problem, all the integrals in equation (9) do exist.

The calculation of $E$ requires the knowledge of $T(r, t)$, which seems to lead a priori to a closed loop. As suggested previously $[1,5]$, we choose the analytical solution of the linear equation corresponding to $\lambda$ and $c$ constants but $\rho=\rho_{0}(1+\alpha T)$, which was given by Hoffer [6].

The solution of equation (7) may be written as :

$$
T(r, t)=\sum_{n=1}^{\infty} T_{n}(r)\left[1-\exp \left(-b_{n} t\right)\right],
$$

where $T_{n}(r)$ and $b_{n}$ are given by :

$$
\begin{gathered}
T_{n}(r)=\frac{-2 \varepsilon \mathrm{a} \kappa J_{0}\left(\xi_{n} r / r_{0}\right)}{b_{n}\left\{J_{0}\left(\xi_{n}\right)\left[\varepsilon-\xi_{n}^{2} \frac{\lambda_{\mathrm{op}}}{r_{0}}\right]-J_{1}\left(\xi_{n}\right)\left[\varepsilon+\frac{\lambda_{\mathrm{op}}}{r_{0}}\right] \xi_{n}\right\}}, \\
b_{n}=\left[\frac{\xi_{n}^{2}}{r_{0}^{2}}\left(1+\alpha^{\prime} E\right)-a \alpha\right] \kappa,
\end{gathered}
$$


where $a=j^{2} \rho_{0} / \lambda_{0}\left[\mathrm{~km}^{-2}\right], \kappa=\lambda_{0} / \delta_{0} c_{0}\left[\mathrm{~m}^{2} \mathrm{~s}^{-1}\right]$ and $\xi_{n}$ are the roats of the following equation [6] :

$$
J_{0}(\xi)-\frac{\lambda_{\mathrm{op}}}{\varepsilon r_{0}} \xi J_{1}(\xi)=0,
$$

$J_{0}(\xi)$ and $J_{1}(\xi)$ are the Bessel functions of the first kind.

2.2 THE CASE WHERE $\lambda(T)=\lambda_{0}$ AND $c(T)=$ $c_{0}(1+\beta T)$. - It is known that for usual electrical conductors such as aluminium and Copper, $c(T)$ may show a strong dependence on temperature $[3,5,7]$. Moreover, any variation of the specific heat with temperature leads to a non-linear heat transfer equation which may be solved within the optimal linearization scheme that we described earlier. In the frequent cases of a linear variation of the specific heat, $c(T)=c_{0}(1+\beta T)$, i.e., the time dependent heat transfer equation becomes :

$$
\frac{\partial^{2} T}{\partial r^{2}}+\frac{1}{r} \frac{\partial T}{\partial r}-\gamma_{0}(1+\beta T) \frac{\partial T}{\partial t}=-a(1+\alpha T)
$$

where $\gamma_{0}=\frac{\delta_{0} c_{0}}{\lambda_{0}}\left[\mathrm{sm}^{-2}\right]$ is the reciprocal diffusivity. The optimal linearized equation corresponding to this case is :

$$
\frac{\partial^{2} T}{\partial r^{2}}+\frac{1}{r} \frac{\partial T}{\partial r}-\gamma_{\mathrm{op}} \frac{\partial T}{\partial t}=-a(1+\alpha T)
$$

where $\gamma_{\mathrm{op}}$ is obtained as :

$$
\begin{aligned}
& \gamma_{\text {op }}=\gamma_{0}(1+\beta F), \\
& F=\frac{\int_{0}^{r_{0}} \int_{0}^{t_{1}} \mathrm{~d} r \mathrm{~d} t T\left(\frac{\partial T}{\partial t}\right)^{2}}{\int_{0}^{r_{0}} \int_{0}^{t_{1}} \mathrm{~d} r \mathrm{~d} t\left(\frac{\partial T}{\partial t}\right)^{2}} .
\end{aligned}
$$

The solution of equation (15) takes the form :

$$
T(r, t)=\sum_{n=1}^{\infty} T_{n}^{\prime}(r)\left[1-\exp \left(-b_{n}^{\prime} t\right)\right]
$$

with

$$
\begin{gathered}
T_{n}^{\prime}(r)=\frac{-[2 \varepsilon a \kappa /(1+\beta F)] J_{0}\left(\xi_{n}^{\prime} \frac{r}{r_{0}}\right)}{b_{n}^{\prime}\left\{J_{0}\left(\xi_{n}^{\prime}\right)\left[\varepsilon-\xi_{n}^{\prime 2} \frac{\lambda_{0}}{r_{0}}\right]-J_{1}\left(\xi_{n}^{\prime}\right)\left[\varepsilon+\frac{\lambda_{0}}{r_{0}}\right] \xi_{n}^{\prime}\right\}}, \\
b_{n}^{\prime}=\left(\frac{\xi_{n}^{\prime 2}}{r_{0}^{2}}-a \alpha\right) \frac{\kappa}{1+\beta F},
\end{gathered}
$$

$\xi_{n}^{\prime}$ are the roots of equation:

$$
J_{0}\left(\xi^{\prime}\right)-\frac{\lambda_{0}}{\varepsilon r_{0}} \xi^{\prime} J_{1}\left(\xi^{\prime}\right)=0 .
$$

\section{Results}

As we have pointed out earlier, we solve numerically the non-linear heat transfer equation in both cases described in sections (i) and (ii). To illustrate this procedure, we consider the first case where $\lambda(T)=$ $\lambda_{0}\left(1+\alpha^{\prime} T\right)$ and $c=c_{0}$. We split the time range from 0 to $t_{1}$ into $N$ intervals equal to $\tau$ (i.e., $N \tau=t_{1}$ ), and obtain :

$$
\begin{gathered}
\frac{\mathrm{d}^{2} T_{i+1}}{\mathrm{~d} r^{2}}=-\frac{1}{r} \frac{\mathrm{d} T_{i+1}}{\mathrm{~d} r}-\frac{\alpha^{\prime}}{1+\alpha^{\prime} T_{i+1}}\left(\frac{\mathrm{d} T_{i+1}}{\mathrm{~d} r}\right)^{2}+ \\
+\frac{\gamma_{0}}{1+\alpha^{\prime} T_{i+1}} \frac{T_{i+1}-T_{i}}{\tau}-\frac{a\left(1+\alpha T_{i+1}\right)}{1+\alpha^{\prime} T_{i+1}},
\end{gathered}
$$

$(i=0,1,2, \ldots, N)$, with the initial condition $T_{0}=0$ for $0 \leqslant r \leqslant r_{0}$. The remaining problem is to solve $N$ equations of this type. This is done by using the Range-Kutta method as in the steady state problem. In this resolution, the boundary conditions of equations (4) and (5) are used. The accuracy of numerical calculation is obviously enhanced when we choose a small time interval $\tau$, or equivalently a large value for $N$. This can be done, however, at the expense of the calculation time which becomes too long. In our case, a compromise between the accuracy desired (of few percents) and a reasonable time required for the calculation is reached when we choose a step $\tau=100 \mathrm{~s}$. We first study the rise of temperature at the centre line $T_{\mathrm{m}}$ as a function of time using the following parameters which characterize the $\mathrm{Al}$ :

$$
\begin{aligned}
\rho_{0} & =2.9 \times 10^{-8}[\Omega \mathrm{m}] \\
\lambda_{0} & =180\left[\mathrm{~W} \mathrm{~m}^{-1} \mathrm{~K}^{-1}\right] \\
c_{0} & =870\left[\mathrm{~J} \mathrm{~kg}^{-1} \mathrm{~K}^{-1}\right] \\
\delta_{0} & =2760\left[\mathrm{~kg} \mathrm{~m}^{-3}\right] \\
\alpha & =43 \times 10^{-4}\left[\mathrm{~K}^{-1}\right] \\
\alpha^{\prime} & =-3.4 \times 10^{-4}\left[\mathrm{~K}^{-1}\right] \\
\beta & =6.68 \times 10^{-4}\left[\mathrm{~K}^{-1}\right] .
\end{aligned}
$$

The other parameters are chosen as :

$$
\begin{aligned}
r_{0} & =5 \times 10^{-3}[\mathrm{~m}] \\
\varepsilon & =16.6\left[\mathrm{~W} \mathrm{~m}^{-2} \mathrm{~K}^{-1}\right] \\
I & =200[\mathrm{~A}] .
\end{aligned}
$$

In realistic cases where $\alpha^{\prime}$ is relatively small, the effects of non-linearity is found to be negligible. This was also the conclusion reached in the steady state problem. Therefore, we decided to choose deliberately a large value for $\alpha^{\prime} E= \pm 0.3$ to enhance the effect of nonlinearity. Although perhaps fictitious, this procedure 
enables us to show that our optimal linearization method provides a powerful tool for solving nonlinear equations. In figure 1 , we have plotted $T_{\mathrm{m}}$ versus $t$ using the data (22) and (23) except $\alpha^{\prime}$ which takes the value $\alpha^{\prime}= \pm 103.8 \times 10^{-4}\left[\mathrm{~K}^{-1}\right]$. The curve 1 represents equation (7) whereas the curve 2 corresponds to the numerical resolution of equation (3) for the same $\alpha^{\prime}$. Interestingly enough, we note that the two optimal curves corresponding to both $\alpha^{\prime} E=+0.3$ and $\alpha^{\prime} E=-0.3$ are almost identical. The same observation is made for the numerical resolution using $\alpha^{\prime}= \pm 103.8 \times 10^{-4}\left[\mathrm{~K}^{-1}\right]$. This is due to the fact that the temperature does not change significantly with $r$. The slight discrepancy between the curves 1 and 2 is mainly due to the choice of $\tau=100$ s. One can verify that the agreement can be improved significantly by choosing a smaller value for $\tau$. This provides a strong argument in favour of the optimal linearization method described here. A remark concerning the choice of $t_{1}$ is in order here. We took $t_{1}=1500 \mathrm{~s}$ to reach an accuracy of the order of :

$$
\frac{T_{\mathrm{m}}-T_{\mathrm{m}}\left(t_{1}\right)}{T_{\mathrm{m}}} \leqslant 10^{-2}
$$

which gives

$$
t_{1} \approx \frac{2 \ln 10}{b_{1}} \approx 1500 \mathrm{~s} .
$$

The curve 3 which is plotted for comparison only, illustrates the case where $\lambda, \rho$ and $c$ are constants.

The effect of the specific heat $c(T)=c_{0}(1+\beta T)$ is illustrated by figure 2 where we used $\beta F= \pm 0.3$. The deviations here are clearly more important than in the previous case. $\beta F=+0.3$ and $\beta F=-0.3$ lead to two different sets of curves which are labelled $a$ and $b$ respectively. But the agreement between the optimal curves and the numerical ones is good and can be improved further by increasing $N$. The case treated above corresponds to a small value for Biot's [8] number $B_{i}=\frac{\varepsilon r_{0}}{\lambda_{0}}\left(\approx 10^{-4}\right)$ which induces as we have observed earlier a small variation of the temperature through the conductor. Although this value of $B_{i}$ corresponds to realistic parameters of aluminium conductors, it may not constitute a good practical example to test our optimal linearization method. We shall choose now conditions in which $B_{i}$ is large by letting [1]

$$
\begin{aligned}
& r_{0}=0.1[\mathrm{~m}] \\
& \varepsilon=2300\left[\mathrm{~W} \mathrm{~m}{ }^{-2} \mathrm{~K}^{-1}\right] \\
& I=290[\mathrm{kA}] .
\end{aligned}
$$

These values correspond to $B_{i}=1.277$ and induce a large variation of the temperature along $r$. They may seem to be too large but they enable us to test correctly our method in extreme conditions. In figure 3 , we have plotted the variation of the temperature at the centre

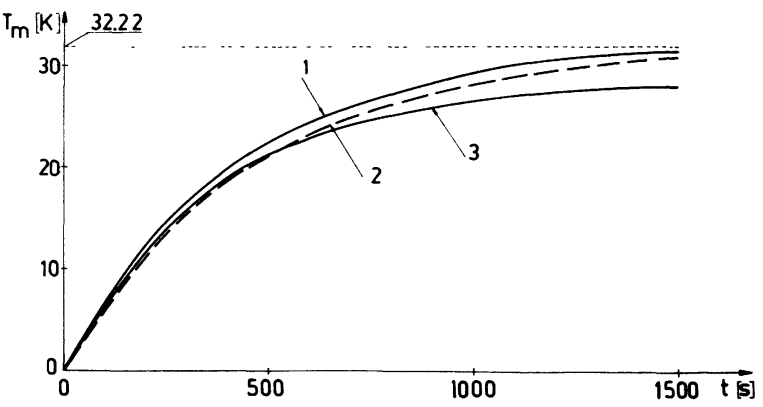

Fig. 1. - The variation of the centre line temperature $T_{\mathrm{m}}[\mathrm{K}]$ with time $t$ [s] for $\mathrm{Al}$ in the case where $\lambda(T)=$ $\lambda_{0}\left(1+\alpha^{\prime} T\right)$, and $B_{i} \approx 10^{-4}$.

Curve 1 represents equation (7) with $\alpha^{\prime} E= \pm 0.3$.

Curve 2 represents equation (3) with $\alpha^{\prime}= \pm 103.8 \times$ $10^{-4} \mathrm{~K}^{-1}$.

Curve 3 represents the case where $\rho=\rho_{0}, \lambda=\lambda_{0}, c=c_{0}$.

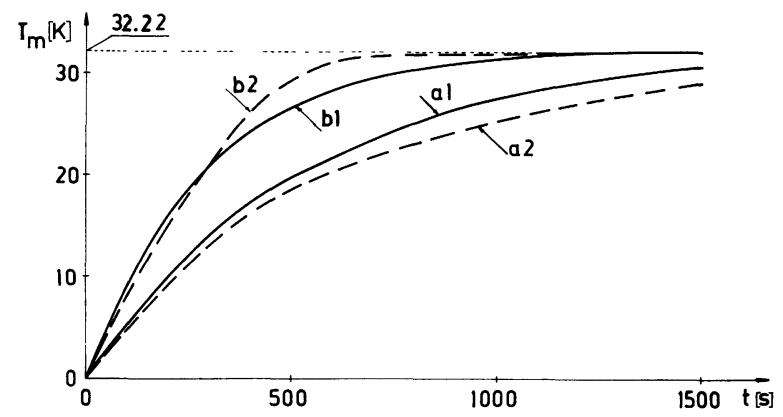

Fig. 2. - The variation of $T_{\mathrm{m}}[\mathrm{K}]$ with time $t$ [s] for $\mathrm{Al}$ in the case where $c(T)=c_{0}(1+\beta T)$, and $B_{i} \approx 10^{-4}$.

Curve (a1) represents equation (15) with $\beta F=+0.3$.

Curve (b1) represents equation (15) with $\beta F=-0.3$.

Curve (a2) represents equation (14) with $\beta=279 \times$ $10^{-4}\left[\mathrm{~K}^{-1}\right]$.

Curve (b2) represents equation (14) with $\beta=-279 \times$ $10^{-4}\left[\mathrm{~K}^{-1}\right]$.

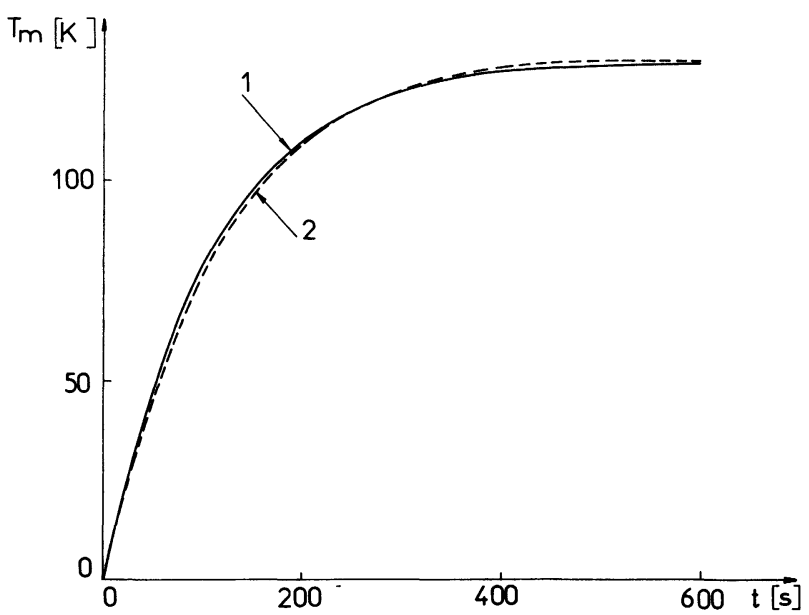

Fig. 3. - The variation of $T_{\mathrm{m}}[\mathrm{K}]$ with time $t$ [s] for $\mathrm{Al}$, in the case where $\lambda(T)=\lambda_{0}\left(1+\alpha^{\prime} T\right)$, and $B_{i}=1.277$. Curve 1 represents equation (7) with $\alpha^{\prime} E=-25.6 \times 10^{-3}$. Curve 2 represents equation (3) with $\alpha^{\prime}=-3.4 \times 10^{-4}\left[\mathrm{~K}^{-1}\right]$. 
line as a function of time $t$, choosing these conditions and obtaining $\alpha^{\prime} E=-25.6 \times 10^{-3}$. We observe that the extreme conditions of this example do not affect significantly the value of $\alpha^{\prime} E$ which remains realistic for aluminium conductors. We also observe that the deviation between the optimal curve and the exact numerical one is very small and do not exceed $1.5 \%$. This illustrates the usefulness of optimal linearization method even in the extreme conditions of the present example. We note that the time step $\tau$ chosen here was $10 \mathrm{~s}$ to improve the numerical accuracy, at the expense of the time required for the calculation which is much higher than in the case of $\tau=100 \mathrm{~s}$.

To show that the temperature drop along $r$ is significant for our example here, we have plotted in figure 4 , the variation of $\Delta T(r)=T(r)-T_{\mathrm{s}}$ as a function of $r$ at different times. One observes that for short time, since the steady state temperature distribution is not reached yet, the discrepancy between the exact numerical results and the optimal ones increases. But, as the time evolves, when we approach the steady state distribution, this discrepancy decreases. In any case, this discrepancy never exceed $2 \%$ at the centre line where the temperature is maximum. One notes that the temperature drop from the centre line to the surface of the conductor is higher than 50 degrees whereas in the previous case of a small value of $B_{i}$, this drop was much less than one degree. This figure, together with the previous one, indicate that the optimal linearization method is good for high values of $\boldsymbol{B}_{i}$.

\section{Conclusions.}

In this paper we apply the optimal linearization method to the resolution of the non-linear, time dependent heat transfer equation. This method provides a powerful tool for solving analytically nonlinear problems. It can be used to describe the dynamical thermal response of the conductor to a constant

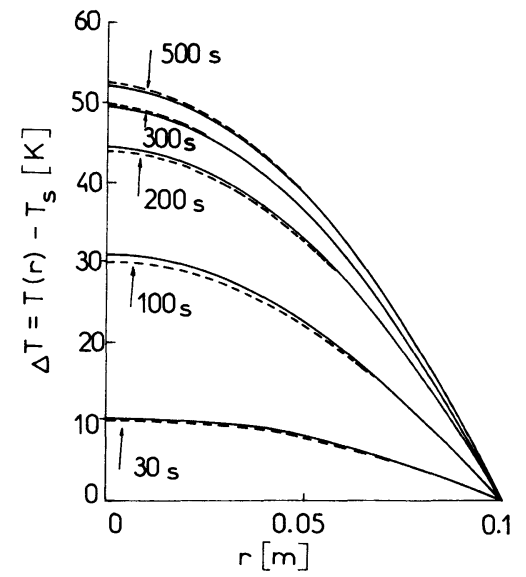

Fig. 4. - The variation of $\Delta T=T(r)-T_{\mathrm{s}}$ as a function of $r$ at different times for $\mathrm{Al}$, in the case where $\lambda(T)=$ $\lambda_{0}\left(1+\alpha^{\prime} T\right)$ and $B_{i}=1.277 . T_{\mathrm{s}}$ is the surface temperature. The dashed lines correspond to the exact numerical calculation, where as the continuous curves correspond to the resolution of the optimal linear equation.

direct current even for a non-linear system. Here we have chosen linear functions for $\lambda(T)$ and $c(T)$ but it is clear that the optimal linearization method can be readily extended to quadratic functions. We have considered both the cases of a very small Biot's number $B_{i}\left(\approx 10^{-4}\right)$ and the case of $B_{i}=1.277$. In the former case, one knows that the effect of non linearity due to $\lambda(T)$ is negligible since it does not lead to a significant drop of temperature from the centre line to the surface of the conductor. However, in the second case of $B_{i}=1.277$, this drop is higher that 50 degrees in our case and yet, the results obtained using the optimal linearization method are very close to the ones obtained by a direct numerical resolution. The comparison of the optimal linearization method and the method of finite elements is under way and the results will be reported elsewhere.

\section{References}

[1] Berbar, B., Jordan, A., Benmouna, M., Steady state temperature distribution in a cylindrical electrical conductor : non-linear effects, Revue Phys. Appl. 18 (1983) 677-681.

[2] LAURENT, M., Etude comparative et critique de quelques méthodes de détermination des caractéristiques thermophysiques des solides conducteurs de la chaleur, Revue Gen. Therm. 238 (1981) 701718.

[3] FARNia, K., BeCK, J. V., Numerical solution of transient heat conduction equation for heat treatable alloys whose thermal properties change with time and temperature, J. Heat Transfer 99 (1977) 471478.

[4] Vujanovic, B., DJukIC, D. J., On one variational principle of Hamilton's type for non linear heat transfer problem, Int. J. Heat Mass Transfer 15 (1972) 1111-1123.

[5] VujanOvic, B., Application of the optimal linearization method to the heat transfer problem, Int. J. Heat Mass Transfer 16 (1973) 1111-1117.

[6] HoFFER, O., Instätionaire Temperaturverteilung in Einem Runddraht, Archiv Elektrotech. 60 (1978) 319-325.

[7] Bransier, J., Caussade, B., Lauriant, J., Jalin, R., BALAGEAS, D., Etude comparative de certaines méthodes de résolution approchée de l'équation de diffusion thermique non linéaire, Entropie 65 (1975) 9-19.

[8] De VRIENDT, A. B., La transmission de la chaleur. Vol 1, Tome 1 (Gaëtan Morin éditeur, Québec) 1982. 